\title{
Multiscale Imaging of HIV-1 Transmission in Humanized Mice
}

\author{
Collin Kieffer, Mark S. Ladinsky, and Pamela J. Bjorkman
}

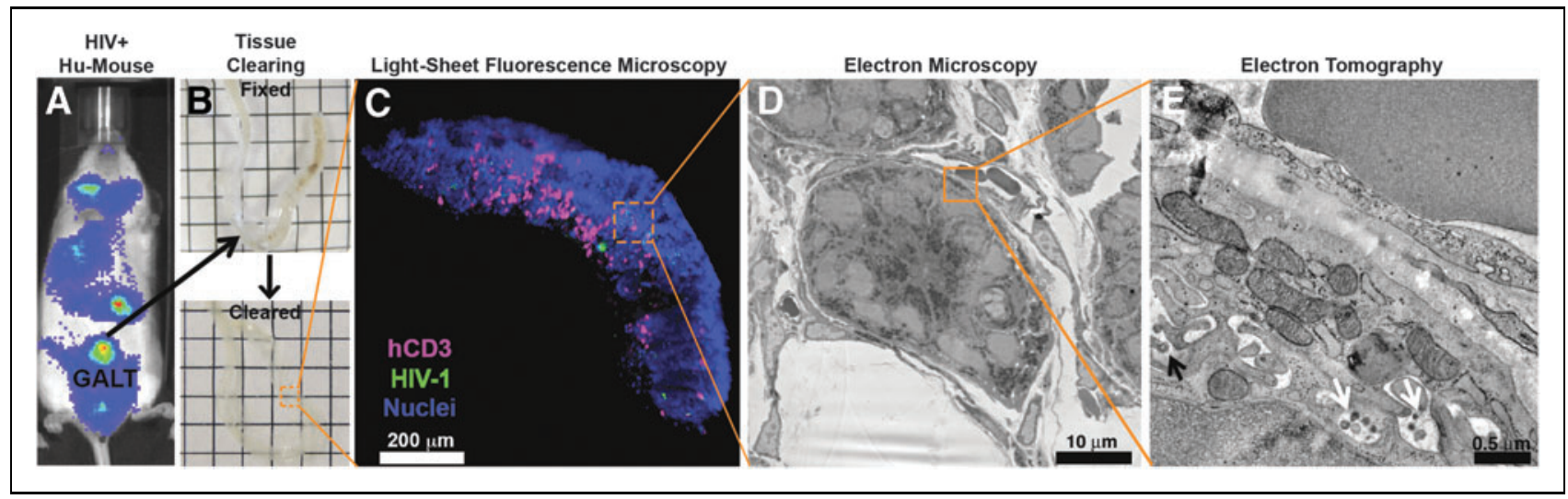

FIG. 1. Despite ongoing efforts to characterize HIV-1 pathogenesis, there remain numerous gaps in our understanding of the biological mechanisms of HIV-1 dissemination in tissues. To visualize the structural details of HIV-1 transmission, we developed a multiscale imaging platform to longitudinally observe virus dissemination at the resolution of individual infected cells and virions in lymphoid tissues from hu-mice. (A) Representative bioluminescence image showing the distribution of human $\mathrm{T}$ cells within a hu-mouse (image courtesy of Rachel Galimidi). Immunodeficient mice (NOD.CgPrkd ${ }^{\text {scid }} I l 2 \mathrm{rg}^{\mathrm{tm} 1 \mathrm{Wjl}} / \mathrm{SzJ}$; NOD/SCID/IL2R $\gamma-/-$; NSG) were injected with stimulated human peripheral blood mononuclear cells to rapidly generate a hu-mouse predominantly reconstituted with activated $\mathrm{T}$ cells. ${ }^{1} \mathrm{Hu}-\mathrm{mice}$ were infected with R5tropic HIV-1 (YU-2), sacrificed at specific times postinfection, and lymphoid tissues including GALT, spleen, and reproductive tract were harvested and fixed for analysis by light and EM. (B) Fixed GALT samples (top) were cleared using the passive CLARITY technique ${ }^{2,3}$ to render tissues visually transparent (bottom) and entire samples were immunostained to identify human cells and viral proteins. ${ }^{1}$ The removal of opaque biomolecules during clearing enhances light penetration into samples, and refractive index matching of tissues, imaging media, and objectives minimizes optical distortions during imaging. These methods were applied to visualize large volumes $\left(\mathrm{mm}^{3}-\mathrm{cm}^{3}\right)$ of intact tissues with single cell resolution using confocal ${ }^{1}$ and LSFM. Dashed box indicates approximate size of region imaged in subsequent panel. (C) 3D-IF image of GALT from an HIV-1-infected hu-mouse captured by LSFM, which utilizes cylindrical lenses to generate thin sheets of light to optically section whole tissues. This method enabled the acquisition of 3D-IF images with single cell resolution and delineated the spatial relationships of HIV-infected cells and target cells within complex tissue environments. Human CD3+ T cells (magenta) were distributed throughout GALT, and HIV-1 p24 staining (green) preferentially localized to colon crypts at the base of villi (dashed box also indicates approximate size of region imaged in ensuing panel). (D) Adjacent regions of HIV-1-infected GALT from hu-mice were prepared for EM by a hybrid method involving high-pressure freezing and freeze-substitution fixation. ${ }^{1,4}$ Thin-section EM overview of a comparable region of colon crypts identified an area (orange box) that contained HIV-1 virions. (E) ET of the virus containing region from (D) revealed 3D ultrastructural information about the localization of individual virions and provided sufficient resolution to distinguish both mature and immature free virions (white arrows) and nascent budding virions (black arrow). Note the orders of magnitude in change of scale between the various imaging methods used to analyze the same sample. We are currently applying these techniques to image additional tissues of interest (i.e., lymph nodes, thymus, brain, and bone marrow) from highly relevant animal models of HIV infection and human clinical samples. Taken together, these efforts highlight the utility of multiscale imaging for understanding both the global pathology and structural details of HIV-1 transmission within lymphoid tissues, and provide new insights for combating HIV spread throughout the body. 3D-IF, 3D-immunofluorescence; EM, electron microscopy; ET, electron tomography; GALT, gut-associated lymphoid tissue; hu-mice, mice with humanized immune system; LSFM, light sheet fluorescence microscopy.

Department of Biology and Biological Engineering, California Institute of Technology, Pasadena, California. 
Keywords: HIV transmission, humanized mice, tissue clearing, light sheet fluorescence microscopy, electron tomography, multiscale imaging

\section{Author Disclosure Statement}

No competing financial interests exist.

\section{References}

1. Kieffer C, Ladinsky MS, Ninh A, Galimidi RP, Bjorkman PJ: Longitudinal imaging of HIV-1 spread in humanized mice with parallel 3D immunofluorescence and electron tomography. Elife 2017;6:pii:e23282.

2. Yang B, Treweek JB, Kulkarni RP, Deverman BE, Chen CK, Lubeck E, Shah S, Cai L, Gradinaru V: Single-cell phenotyping within transparent intact tissue through wholebody clearing. Cell 2014;158:945-958.

3. Treweek JB, Chan KY, Flytzanis NC, Yang B, Deverman BE, Greenbaum A, Lignell A, Xiao C, Cai L, Ladinsky MS, Bjorkman PJ, Fowlkes CC, Gradinaru V: Whole-body tissue stabilization and selective extractions via tissue-hydrogel hybrids for high-resolution intact circuit mapping and phenotyping. Nat Protoc 2015;10:1860-1896.

4. Ladinsky MS, Kieffer C, Olson G, Deruaz M, Vrbanac V, Tager AM, Kwon DS, Bjorkman PJ: Electron tomography of HIV-1 infection in gut-associated lymphoid tissue. PLoS Pathogens 2014;10:e1003899.

Address correspondence to: Pamela J. Bjorkman Department of Biology and Bioengineering, MC 114-96 California Institute of Technology 1200 East California Boulevard Pasadena, CA 91125

E-mail: bjorkman@caltech.edu 